\title{
La revelación en los estados financieros
}

JORGE M. GI**

\section{El modelo contable}

\section{como modelo descriptivo}

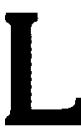

a conceptualización del modelo contable es un tema que está gozando de cierta reputación en los medios académicos vinculados a la contabilidad. La insuficiencia metodológica que presupone considerar el conocimiento contable como exclusivamente técnico, ha incentivado otros enfoques que aspiran a darle a la contabilidad un status epistemológico por lo menos distinto. Dentro de estos enfoques (existen otros que no trataremos en el presente trabajo), y a partir de la divulgación del concepto de modelo en las ciencias administrativas ${ }^{(1)}$, comienza a desarrollarse la tesis que considera a los estados financieros de la empresa como modelo. Siguiendo un razona-

* Profesor Unlversidad Nacional de la Patagonia, San Juan Bosco, Argentina.

(1) Es importante la influencia que han tenido las ciencias administrativas cuando estudian las empresas como modelo de organización, a partir -principalmente de $H$. Simón y su esquema de ciencias artificiales. No obstante, por lo menos en los autores argentinos (Garcla, Lazatti, Fowler Newton, Lopez Santiso, entre otros), quienes mayor incidencia metodológica han tenido en este tema son D.W. Miller y M.K. Star principalmente a través de su libro "Acuerdos ejecutivos de operaciones". miento en extremo lineal, esa corriente del pensamiento contable caracteriza, tanto a los modelos en general como a los estados financieros en particular por sus objetivos. Así, el objetivo de los estados contables es "proporcionar información sobre la situación y la evolución del patrimonio de los entes" (2) y la definición de modelo destaca que se trata de "una representación de la realidad que intenta describir, explicar o predecir cómo se comporta algún aspecto de ella" (3). De la articulación de ambas definiciones, emerge la propuesta básica: "los estados financieros son modelos de la realidad". Sin entrar a analizar la validez conceptual de este enfoque, podemos convenir en que representa un avance sobre el esquema predominantemente técnico y registral que se usaba en Contabilidad.

(2) Fowler Newton, Enrique. "Cuestiones contables fundamentales". Ediciones Contabilidad Moderna, Buenos Aires, 1982.

(3) Miller, D.W. y Start M.K. "Acuerdos ejecutivos de operaciones" citado por Lopez Santiso, H. y otros en "Estados Contables en moneda constante", Ediciones Macchi, Buenos Aires, 1989, pág. 23. 
Los estados financieros de una empresa pueden ser leídos bajo distintos enfoques (según cual fuere el aspecto o dimensión que nos interese del "modelo-estados financieros"):

\section{- Dimensión descriptiva:}

Los informes contables (entre los que definimos a los estados financieros), pueden ser entendidos como un relato emergente de la composición y el origen del patrimonio y los resultados de una empresa. Una descripción no sólo cuantitativa pues las notas, anexos, reportes y memorias contribuyen a incluir los aspectos cualitativos de la actividad de la empresa (política comercial, tecnología productiva, esquema de organización empresarial e, inclusive, algunos aspectos relativos al área de la contabilidad social).

- Dimensión interpretativa:

A partir de la aplicación de las técnicas de análisis sobre los "modelos-estados financieros" es posible encontrar las causas del performance de la empresa, de su situación económica, financiera y patrimonial.

Esta dimensión implica la capacidad del modelo para estructurarse a sí mismo como modelo interpretativo (capaz de mostrar las relaciones de causa-efecto que lo condicionan).

\section{- Dimensión normativa:}

Es la dimensión que se vincula al orden interno del "modelo-estados financieros" y a la lógica de articulación de ese modelo con la realidad.

El primer aspecto (orden interno), se ocupa o se refiere a la posibilidad de mejorar la entropía del modelo (por ejemplo, los criterios de exposición en los distintos estados financieros). La lógica de articulación se vincula con las disposiciones legales o imperativas que operan como requisitos preexistentes al modelo (son ejemplo de ello, las leyes, decretos, resoluciones y recomendaciones, sean de origen estatal o convencional).

En todas las dimensiones analizadas, el modelo opera como vehículo de transferen cia entre la realidad y el conocimiento de la misma, a través de la característica esencial de un modelo: transmitir información.

Por supuesto, un modelo permite una variante extensa de operaciones que pueden concretarse con él o a través de él.

Podemos, por ejemplo, predecir el comportamiento futuro del modelo si las variables de entrada sufren modificaciones (esta estrategia es muy adecuada para la formulación de pronósticos). También podemos usar al propio modelo para analizar su sensibilidad (esta posibilidad se utiliza para la selección de caminos alternativos en el proceso de toma de decisiones).

Pero, de todas las posibilidades instrumentales respecto del uso del "modeloestados financieros", sólo vamos a ocuparnos de un aspecto: los "modelosestados financieros" como información. $O$, si se prefiere, los aspectos semióticos del modelo contable. (Esto quiere decir que no estudiaremos las relaciones causaefecto que se derivan del enfoque 
interpretativo. Sí, dedicaremos algún espacio a los aspectos normativos del modelo).

\section{1) El sistema contable como estructurante del modelo contable}

Si bien los modelos son -de por sí- un sistema y operan como tales, en el caso particular de la contabilidad podemos referimos a los estados financieros como exteriorizaciones concretas de la producción del sistema contable. Esto implica admitir que no existe "modeloestados financieros" sin existencia de sistema contable y que aquellos son uno de los productos posibles del sistema (que produce también control, registraciones y otra variada gama de informaciones). Por supuesto que la eficiencia del modelo dependerá de la calidad del sistema contable. Podemos afirmar que el sistema de contabilidad de un ente es -parafraseando a Francia (4). “...un continuo y limitado complejo o conjunto de partes, elementos, componentes, variables, procesos, objetos, atributos o factores-todos denominados subsistemasen mutua interacción y ordenados dinámicamente, durante un período de tiempo determinado".

Así podemos distinguir dentro del sistema contable, una serie de subsistemas tales como:

- El subsistema de organización del trabajo (organigrama contable de tareas, controles y responsabilidades vinculadas a las registraciones $y$

(4) Francia, Alvaro. "Introducción a la teoría general de sistemas", Biblioteca Mosaico, Buenos Aires, 1984, pág. 65. producción de control e información del ente).

- El subsistema del proceso contable (fluxograma y esquema del procedimiento vinculado tanto a la captación, como al control, análisis y registro de los datos).

- El subsistema de control (control interno, auditoría de estados financieros y control de gestión administrativa).

Cada uno de los subsistemas implica una serie de elementos concretos que asumen la forma de bienes físicos (medios de registro y procesamiento como máquinas de contabilidad, computadoras), documentación administrativa (comprobantes de transacciones, asientos de registro, planes y manuales de contabilidad) y registros de la información financiera (libros, fichas).

Estos elementos se complementan con un esquema de interacción y ordenamiento que dan consistencia al sistema global y que constituyen su propia lógica de articulación:

- Los algoritmos que hacen operable el sistema de partidas contables (sean los algoritmos simples de la partida doble: débitos, créditos y saldos o los de mayor complejidad que emergen de la contabilidad matricial: matrices $y$ vectores).

- Los mecanismos de control (de cada subsistema, inclusive el de los estados financieros como producto final del mismo).

En esta visión sistemática importa reconocer: 
- Que es preciso otorgar a la problemática contable una conceptualización más integral y abarcativa que la tradicional. En ese sentido, los "modelos-estados financieros" deben satisfacer su propia condición de modelos de la realidad económica. Ello importa reconocer carácter general a los objetivos de la información, en contraposición con la teoría prevalente respecto de la utilidad sectorial de tal información (por ejemplo: la tradicional postura sobre que los estados contables deben satisfacer los intereses de accionistas, de acreedores o de la propia dirección de la empresa).

Por supuesto que este criterio opera respecto de los estados financieros como modelo, pero no es necesariamente aplicable a toda la información económica utilizada en el proceso de toma de decisiones. En tal caso, puede ser el interés del propio decididor el que actúe como directriz de la información contable. (Cabría distinguir entre el producto del sistema contable destinado a ser presentado a terceros $\mathrm{y}$ aquel que tiene uso interno. Este último, que suele denominarse contabilidad gerencial, es consecuencia de la aplicación de un criterio finalista a la información contable. La cual se adecúa a la necesidad del proceso de toma de decisiones).

- Que el comportamiento tanto como las propiedades de cada subsistema inciden en el sistema, el que a su vez tiene propiedades y un comportamiento propio cuyos efectos se hacen sentir en el sistema. La propia experiencia profesional nos permitiría a cada uno de nosotros reconocer esta circunstan- cia. ¿Cuántas veces el subsistema de organización no resistió el impacto de una modificación en los medios de registro, por ejemplo, la instalación de un sistema de PED?

¿Quién no vivió alguna experiencia de insuficiencias del subsistema de procesos contables frente a requerimientos de control? Y la incapacidad (y necesidad de modificación) de éstos por fallas, errores o fraudes de la organización del trabajo.

En estos casos, pensemos si el sistema contable -en su conjunto- restableció el equilibrio y vamos a advertir que no fue así y que fue preciso incluir novedades en el propio sistema. De allí podemos observar que el sistema contable se encuentra condicionado por el subsistema más inestable. Nos animaríamos a proponer, como tesis, que el subsistema más inestable es el de la organización del trabajo, lo que debería actuar como potenciador de la formación de recursos humanos por parte de las Escuelas y Facultades de Contaduría Pública de América Latina, dentro de un marco de desarrollo científico de la contabilidad.

- Que existen ciertas propiedades de los sistemas ${ }^{(5)}$ que es preciso referenciar al sistema contable:

a) El funcionamiento o comportamiento de cada subsistema afecta el funcionamiento o comportamiento del sistema en su conjunto: así, por ejemplo, la extemporaneidad, demora o insuficiencia del subsistema del proceso contable puede originar

(5) Francia, Alvaro, obra citada en (4), pág. 53. 
desutilidad del "modelo-estados financieros" por falta de oportunidad. La extemporaneidad en los registros, se vincula con el criterio de devengamiento y puede ser -por ejemplo-la falta de asiento de la parte proporcional mensual del denominado sueldo anual complementario. La demora se relaciona más bien con la lentitud operativa del sistema contable, principalmente en la etapa de captación de datos. La insuficiencia se refiere a la falta de integralidad en el relevamiento de todos los datos de la operación. $\mathrm{Si}$-por ejemplo- no se segregan los componentes financieros implícitos incluidos en una venta, la información sobre la transacción que se revela, no es la real).

b) Ningún subsistema tiene un efecto independiente sobre todo el sistema, ya que aquel siempre actúa interrelacionado con algunos otros subsistemas, formando subgrupos. Por ejemplo, el grupo "plan de cuentas-control interno", como elementos interrelacionados de dos subsistemas. Una modificación del plan de cuentas (subsistema del proceso contable) no tiene un efecto independiente sobre el sistema contable, sin -previamente-alterar la división del trabajo de imputación (subsistema de control). La decisión de aperturar cuentas de movimiento para descentralizar el registro de las ventas de cada "caja" en un supermer cado, por ejemplo, implicaría:

- Modificar un elemento del subsistema de registro contable (incluir las cuentas "Recaudaciones por ventas Caja Nro..." en el plan de cuentas).

- Alterar el sistema de control primario (establecer un régimen de arqueo por caja).

- Modificar otro elemento del subsistemade registrocontable (determinar un procedimiento de consolidación de las recaudaciones).

- Alterar el sistema de control secundario (fijar pautas para verificar el depósito bancario del total consolidado). Yasísucesivamente.

Todo ésto nos permite afirmar que, tanto el comportamiento (en el sentido de reacción) como las propiedades (en el sentido de las cualidades) de los subsistemas, inciden en el sistema, el que a su vez tiene propiedades y un comportamiento propio cuyos efectos se hacen sentir en cada subsistema. La sanción de una ley que determine una innovación de registro y exposición (por ejemplo, la obligatoriedad del Estado de Origen y Aplicación de Fondos), opera sobre el sistema contable en cuanto incorpora la necesidad de una nueva información especializada. Ello puede reformular esquemas y procedimientos en el resto de los subsistemas: nuevos cursos en el manual y plan de cuentas, adaptación del organigrama contable por aparición de una nueva función y establecimiento de controles financieros sobre rubros específicos.

La lógica del sistema contable tal como lo hemos estado describiendo, no parece ser la lógica de la información, como han venido sosteniendo hasta ahora los PCGA, sino la lógica de realidad social. La lógica de la información es una lógica finalista, donde privan los intereses 
particulares de los usuarios.

Son éstos quienes discriminan -a partir de su propia utilidad- cómo se organiza la información.

"A partir de mis propios intereses, yo decido la organización de la lectura del periódico tanto como de un estado contable y -es más- deseo que los estados financieros organicen la información de esta manera": este es el apotegma de la lógica de la información. A contrario de ese razonamiento, la lógica de la realidad social, plantea:

- La inutilidad del enfoque de "modelo de información", por constituir una tautología y considerando que la cualidad informativa es esencial al modelo.

- La organización de la información contable como revelación de la realidad.

\section{2) Modelo contable e información}

Al negar la lógica de la información como elemento estructurante del sistema contable, no sólo nos alejamos de la definición instrumentalista de la contabilidad ("...es un sistema de información que debe proporcionar datos posibles para facilitar la toma de decisiones, generalmente vinculados con los negocios" (6), sino de la esencia misma de ese razonamiento que mantiene que "...la información contable no es inmanente sino trascendente. Esto es, no vale por sí, sino por la utilidad de su uso" ${ }^{(7)}$.

\footnotetext{
(6) Cita (3), pág. 51.

(7) Cita (3), pág. 51 .
}

Debemos destacar que esa definición -que podrían compartir algunos científicos de primer nivel como Bonsack (8)- es traída a la materia contable forzando su ámbito y sin previamente conceptualizar el conocimiento contable. El hecho de que un conocimiento produzca información ${ }^{(9)}$ no habilita a suponer que la teoría de la información explica e ilumina esa rama del conocimiento. En sí, todo conocimiento produce información y los "modelos-estados financieros" son estructuras que se transfieren desde la realidad hasta su uso en el proceso de toma de decisiones. "Una información, en sí, no es nada, no tiene nada de información si nadie es informado o si la información recibida no es utilizable para el que la recibió" dice Bonsack (10) y esta definición entra en inmediata contradicción con la adoptada respecto de "modelo".

Nos queda entonces en que, si es modelo, es más que información y, si sólo es información, no podríamos definirlos como modelos.

Los estados financieros son información, tanto como el organigrama o la fórmula técnica de producción de una industria. Lo que hace la información es transformar en comunicable al "modelo-estados financieros" como estructura emergente de la realidad económica de una empresa.

(8) Se refiere a Francois Bonsack.

(9) "Una finalidad básica de la ciencia, en tanto constituye una forma de conocimiento, es brindar información", según lo expresan $\mathbf{R}$. Gaeta y $\mathbf{N}$. Robles en "Nociones de epistemología", EUDEBA, Buenos Aires, 1986, pág. 14.

(10) Bonsack, F, en "Es objetable y matematizable la información?", Coloquios de Royaumont, Siglo XXI Editores, 6a. Edición. México, 1982, p. 223. 
Nuestra propuesta metodológica es:

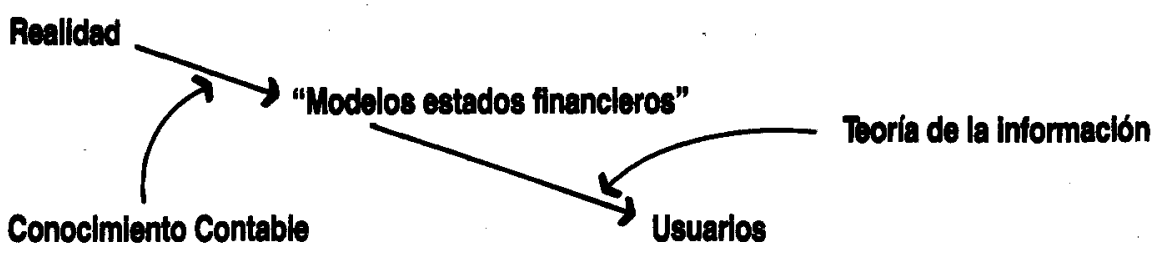

Proponemos entonces la siguiente defi- a códigos que transforman la realidad nición: en información contable útil para la toma de decisiones.

Los "modelos-estados financieros" son las estructuras que -a partir del uso Es preciso tomar en cuenta que los sistemático del conocimiento contable- "modelos-estados financieros" operan permiten transferir la realidad, mediante como variables de entrada en el proceso un proceso denominado revelación finan- decisorio. Veamos el esquema básico ciera. Esa revelación se efectúa en base de funcionamiento del modelo:

\begin{tabular}{|c|c|c|}
\hline VARIABLES DE ENTRADA & SISTEMA & VARIABLES DE SALIDA \\
\hline $\begin{array}{r}\text { CONTEXTO } \\
\text { SOCIOECONOMICO } \\
\text { TRANSACCIONES } \\
\text { EMPRESARIAS }\end{array}$ & $\begin{array}{l}\text { ORGANIZACION } \\
\text { ALGORITMOS } \\
\text { PROCESO } \\
\text { CONTABLE } \\
\text { MECANISMOS } \\
\text { DE CONTROL }\end{array}$ & $\begin{array}{l}\text { MODELOS } \\
\text { ESTADOS } \\
\text { FINANCIEROS } \\
\text { INFORMACION } \\
\text { CONTROL }\end{array}$ \\
\hline
\end{tabular}


Mientras que el esquema de toma de decisiones ${ }^{(11)}$ podría simplificarse en:

\begin{tabular}{|c|c|c|}
\hline VARIABLES DE ENTRADA & SISTEMA & VARIABLES DE SALDA \\
\hline $\begin{array}{r}\text { MODELOS-ESTADOS } \\
\text { FINANCIEROS } \\
\text { INFORMACION }\end{array}$ & $\begin{array}{l}\text { ANALISIS } \\
\text { ALTERNATIVAS } \\
\text { INTERPRETACION } \\
\text { OBJETIVOS } \\
\text { PROYECCION }\end{array}$ & DECISION \\
\hline
\end{tabular}

Compartimos plenamente la idea de subsumir los "modelos-estados financieros" como información estructurante del proceso de toma de decisiones. Pero estaríamos allí en otro estadio, en otro nivel del análisis. Si el campo de la contabilidad fuera el sistema de toma de decisiones, no dudaríamos un minuto en admitir metodológicamente el esquema de la teoría de la información. Pero el campo de nuestra ciencia es mucho más que eso. En alguna circunstancia (12) decíamos "...el replanteo del conocimiento contable podría pasar por el establecimiento de una teoría métrica de la contabilidad, así como la teoría de los objetivos del sistema económico. La teoría métrica debería desarrollar el concepto de unidad de cuenta, tanto desde el punto de vista convencional

(1I) Nos inspiramos en Herbert Simon, "Administración de Empresas en la era electrónica", Editorial Letras S.A., México, 1963, citado por López Santiso y otros, obra citada en ${ }^{(3)}$, pág. 6 . como científico y, no necesariamente monetario.

También esta teoría debiera avanzar sobre los criterios de medición, tanto de la realidad como del valor emergente de las transacciones económicas. Debe también precisar su propio límite, jerarquizando la medición de lo que puede ser medible y desarrollando algunas formas de exposición sobre lo no medible pero importante para describir la realidad.

La teoría de los objetivos del sistema económico debe plantear el origen de la organización de la producción, distinguiendo lo económico de lo financiero; la utilización de la inversión, de su productividad; el proceso de creación del valor, del apropiamiento del excedente o la imputación del déficit".

(12) El autor se refiere a su participación en el Octavo Congreso del Colegio Colombiano de Contadores Públicos. Memorias del Congreso Editorial Legis, Pág. 27. 
En definitiva, el conocimiento contable se ocupa del patrimonio. Esto nos lleva a la tesis de la dinámica del patrimonio (que tratamos como derivados de los objetivos del sistema económico) y al establecimiento de una teoría métrica de la contabilidad (unidad de cuenta, valuación).

Cuando reflejamos un asiento, es porque queremos describir una transformación patrimonial (un hecho económico, administrativo, legal, jurídico que se vincula con la administración empresaria) bien sea esencial (por ejemplo, el registro de una venta) bien sea previo, complementario o derivado de la transformación patrimonial (por ejemplo, una contingencia). Sólo podemos entender la realidad patrimonial (y consecuentemente su dinámica administrativa, financiera $\mathrm{y}$ económica) a través de la Contabilidad. $Y$ es a través de ella que modelamos (estructuramos) esa realidad patrimonial. Lo que discutimos es que la lógica de la modelación no es la de la información, porque el producto del conocimiento contable es primero modelo y -en tanto tal- es información.

\section{3) La lógica y el lenguaje contable}

Un debate sobre la lógica contable, nos trasladaría a la tiniebla de los tiempos.

El jerarquizado investigador Dr. Richard Mattesich (13) ilumina los orígenes de la

(13) El autor se refiere al artículo "Counting, Accounting, and the imput-output principle: recent archeological evidence. Revising our view on the evolution of early record keeping", distinguido como "Best 1988 paper" en La Convención Anual de la Canadian Academie Accounting Association en Windsor, Ontario. La contabilidad a partir de las investigaciones arqueológicas en el Medio Oriente efectuadas por la prof. SchmandtBesserat y nos dice "La conclusión más importante es que aquellos hombres (se refiere a los habitantes de la zona, 8000 años A.C.) fueron los primeros en aplicar el principio de entrada-salida (inherente a cada transacción de bienes muebles) a un sistema de registro de datos representacional, casi numérico (y posteriormente numérico).

Por encima de todo ésto, se demuestra que esta manifestación del principio de entrada-salida refleja la misma estructura lógica del sistema de partida doble...".

Mattesich mantiene que el fundamento de la contabilidad no debe buscarse en las técnicas de la partida doble sino en la forma lógica de una transacción. Por tanto -decimos nosotros- no puede pensarse en la lógica instrumental del registro por partida doble como sustratum del razonamiento contable.

La lógica contable es la lógica de un lenguaje que permite comunicar a través de signos convencionales y mediante una estructura predeterminada, la realidad económica del patrimonio de una organización. Los signos convencionales están dados por conceptos (nivel cualitativo) y mediciones de ese concepto (nivel cuantitativo) ${ }^{(14)}$. La estructura de organización de esos conceptos permite articularlos entre sí en función de su especialización (Todos los signos que se relacio-

traducción al español de ese excelente trabajo, será publicada en el Nro. 7 de Teuken Revista de Investigación Contable (Universidad Nacional de la Patagonia, Argentina). 
nen con el patrimonio, confluirán en el Estado de Situación Patrimonial; los que revelan la actividad económica, en el Estado de Resultados; los que vinculen al funcionamiento se integrarán en el Estado de Origen y Aplicación de Fondos; los que revelen su esencia monetaria en el Estado de Variaciones de Capital Monetario Neto).

Uno de los principales requisitos del signo (por lo menos para la conceptualización contable) es su simetría y su economía. Decimos que un signo contable es simétrico con la realidad cuando permite reconstruirla, mediante un proceso lógico de inferencia. Decimos que un signo contable es económico cuando sintetiza la mayor cantidad de información sobre algún aspecto del patrimonio o de su administración.

Como toda tecnología, la contable... "encuentra en el lenguaje un vehículo esencial y una prueba de ello es la creación de lenguajes específicos en cada disciplina y su enriquecimiento permanente, no sólo en la que se refiere al vocabulario, sino también a la propia estructura lingüística, y no puede ser una casualidad que las ciencias más avanzadas hagan una copiosa utilización de un gran aparato simbólico" (15). Así, por ejemplo, el "Activo igual a 500.000 pesos" es una expresión lingüística que sólo cumple una función informativa cuando tiene sentido decir de ella que es o bien verdadera o bien falsa. Existe una equivalencia lógica que vincula al "Activo" con la realidad (el principio de simetría del que hablamos más arriba). La conceptualización de identidad a través del signo "igual" en el sentido de

(15) Texio citado en ${ }^{(9)}$, pág. 56. equivalente. $Y$ por último, el resultado de la medición: cantidad y unidad de medida ("500.000 pesos").

Veamos que cada término contable encierra una convención. "Activo" -por ejemplo-es una convención legal, jurídica, que se fundamenta en el esquema de apropiación vigente en el sistema económico y que adopta -en la estructura de la contabilidad como lenguaje- una significación específica.

La especificidad de la lingüística contable pasa a transformarse en condición del "modelo-estados financieros" y a partir de este hecho, tanto la terminología como el propio modelo tienden a transformarse en esotéricos (en el sentido de reservado exclusivamente a quienes manejan cierto herramental de conceptos, hipótesis y teorías). Uno de los riesgos en la evaluación de los signos contables -cuando no se encuentran articulados a una teoría general factible de ser axiomatizada- es que se vuelvan asimétricos (alejándose de la realidad que debieran reflejar) y extremadamente complejos en su interpretación.

Un ejemplo concreto del obstáculo (en la definición de Bachelard) que produce al conocimiento contable la ausencia de desarrollos teóricos que fundamenten la propia lógica de la disciplina, la constituye el estudio de los problemas que planteó -en Argentina- el reconocimiento contable del impacto de la inflación en el patrimonio y su repercusión en los "modelos-estados financieros" (16).

(16) Gil, Jorge M., "Cincuenta años de inflación y su reconocimiento contable en la Argentina", en prensa, Madrid. España. 
Por ello, debemos estructurar la teoría contable a partir de la lógica usada en las ciencias artificiales (en el sentido de nota (1) reconociendo que los "modelosestados financieros" son algo más que información: constituyen objetos sobre los que operamos para conocer la realidad y -recién entonces- tomar decisiones. Desde el punto de vista epistemológico, el conocimiento contable exige la formación del esquema mental artificial, internalizando la estructura lógicomatemática, pero -a partir de allí- se fundamenta en la realidad social (17).

Reiteramos entonces que, si la lógica contable es la emergente de su simetría con la realidad, la construcción de modelos (entre ellos los "modelosestados financieros"), no puede estructurarse exclusivamente sobre convenciones sociales (provengan de la legislación positiva, como una ley, o se originen en normas emitidas por los organismos profesionales).

Compartimos la posición de Cañibano (18) quien opina que "el método contable es un conjunto de postulados e hipótesis... que permiten pasar de la realidad económica a los estados contables que informan sobre la misma" entre los que se destacan:

“a) Principio de dualidad, eje básico sobre el que descansa la observación contable de la realidad económica, a cuyo través se efectúa la captación de los datos correspondientes.

b) Medición y valoración, conjunto de reglas alternativas que permiten la expresión cuantitativa de los fenómenos económicos con un determinado grado de objetividad.

c) Formas de representación, como expresión de las diferentes aplicaciones de registrar en un lenguaje contable los sucesos económicos.

d) Agregación o conjunto de normas que permitan el procesamiento de datos con fines informativos, haciendo posible la obtención de estados de síntesis que contengan agregados económicos relevantes".

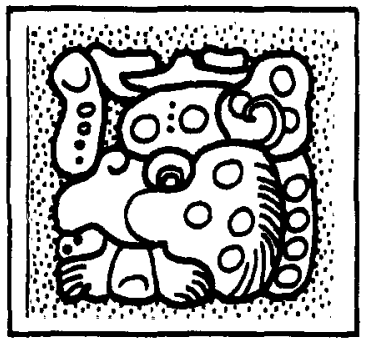

(17) Gil, Jorge M. y otros, "El método cualitativo de investigación aplicado a la Contabilidad", Revista de Investigación Contable TEUKEN, Nro. 0, pág. 7-18, Comodoro Rivadavia, Argentina, enero de 1988.
(18) Cañibano, Leandro, "Contabilidad: Análisis contable de la realidad económica", Editorial Pirámide, España, 1987, pág. 53. 


\section{Bibliografias}

\section{El papel de la Teoría Contable}

AMERICAN INSTITUTE OF CERTIFIED PUBLIC ACCOUNTANTS, Objectives of Financial Statements (Trueblood Report). Report of the Study Group on the Objectives of Financial Statements. New York: AICPA, 1973.

ARROW, K.J., Social Choice and Individual Values, Cowles Foundation Monograph. New York: John Wiley, 1963.

R.J. And P. Brown, “An Empirical Evaluation of Accounting Income Numbers”, Journal of Accounting Research 6 (Autumn 1968), pp. 159-178.

BEAVER, W.H., "Financial Ratios as Predictors of Failure", Empirical Research in Accounting: Selected Studies 1966, supplement to Vol, 4 of Journal of Accounting Research (1966), pp. 71-111.

1981.

BENSTON, G.J., Published Corporate Accounting Data and Stock Prices", Empirical Research in Accounting: Selected Studies 1967, supplement to Vol. 5 of Journal of Accounting Research (1967), pp. 1-14 and 22-54.

BLAUG, M., The Methodology of Economics. Cambridge University Press, 1980.

CHAMBERS, R.J., Accounting, Evaluation and Economic Behavior. Englewood Cliffs, N.J.: Prentice-Hall, 1966.

DYCKMAN, T.R., And S.A. Seff, "Two Decades of the Journal of Accounting Research: Journal of Accounting Research 22 (Spring 1984), pp. 225-297.

EDWARDS, E.O., And P.W. Bell, The theory and Measurement of Business Income. Berkeley: University of California Press, 1961.

FRIEDMAN, M., "The Methodology of Positive Economics", Essays in Positive Economics. Chicago: University of Chicago Press, 1953, reimpreso por Chicago: Phoenix Books, 1966.

PASSMORE, J.A., "Can the Social Sciences Be Value-Free?" in H. Feigl and M. Brodbeck, eds., Readings in the Philosophy of Science, pp. 674-676. New York: Appleton-Century-Crofts, 1953, pp. 674-676.

POPPER, K.R., Conjectures and Refutations: The Growth of Scientific Knowledge. London: Routledge \& Kegan Paul, 1963.

New York: Harper Torch Books, 1965. 


\section{Posibilidad y utilidad de la Teoría Positiva de la Contabilidad}

Auerbach, J.S., Enmity and Amity: Law Teachers and Practitioners, 1901)-1922, Perspectives in American History (1971) pp. 551-601.

Bhaskar, R., A Realist Theory of Science (Leeds: Leeds Books, 1975).

Bhaskar, R., The Possibility of Naturalism (Brighton: Harvester, 1979).

Blaug, M., The Methodology of Economics (Cambridge: Cambridge University Press, 1980).

Bledstein, B.J., The Culture of Professionalism (New York: Norton, 1976).

Caldwell, B., Beyond Positivism: Economic Methodology in the Twentieth Century (London: Allen and Unwin, 1982).

Calvert, M.A., The Mechanical Engineer in America, 1830-1900 (Baltimore: John Hopkins University Press, 1967).

Chandler, A.D., The Visible Hand (Cambridge, MA: Harvard University Press, 1977).

Chandler, A.D., Historical Determinants of Management Hierarchies en Van de Ven, A.H. and Joyce, W.F. (eds.) Perspectives on Organization Design and Behaviour, (New York: John Wiley, 1981).

Channell, D.F., The Harmony of Theory and Practice: The Engineering Science of W.J.M. Rankine, Technology and Culture (1982), pp. 39-52.

Christenson, C., The Methodology of Positive Accounting, The Accounting Review (1983) pp. 1-22.

Coats, A.W., Introduction, in Coats A.W. (ed.) Economists in Government (Durham, NC: Duke University Press, 1981).

Coddington, A., Positive Economics, Canadian Journal of Economics (1972) pp. 1-15.

Collins, H., The Seven Sexes: A Study in the Sociology of a Phenomenon or the Replication of Experiments in Physics, Sociology (1975) pp. 205-224.

Collins, H. (ed,), Knowledge and Controversy: Studies of Modern Natural Science, special issue of Social Studies of Science (1981), pp. 1-158.

Daele, W. v.d., The Social Construction of Science en Mendelsohn, E, et al, (eds.) The Social Production of Scientific Knowledge, Sociology of the Sciences Yearbook 1, (Dordrecht: Reidel, 1977).

Daems, H., The Determinants of the Hierarchical Organization of Industry, en Francis A, et al. (eds.) Power, Efficiency and Institutions (London: Heinemann, 1983).

Deane, P., The Scope and Method of Economic Science, Economic Journal'(1983) pp. 1-12.

Dyckman, T.R. \& Zeff, S.A., Two Decades of the Journal of Accounting Research, Journal of Accounting Research (1984) pp. 225-297.

Eichner, A.S., Why Economics is Not Yet a Science, en Eichner, A.S. (ed.) Why Economics is Not Yet a Science (London: Macmillan, 1983).

Farley, J. \& Geison G.L., Science Politics and Spontaneous Generation in Nineteenth Century France: The Pasteur-Pouchet Debate, en Chant, C. and Fauvel, J. (eds.) Darwin to Einstein: Historical Studies on Science and Belief (London: Longman, 1980). 
Fay, Brian, Social Theory and Political Practice (London: Allen and Unwin, 1975).

Feyerabend, Paul K., Problems of Empiricism, en Colodny R. (ed.) Beyond the Edge of Certainty, (Englewood Cliffs, NJ: Prentice-Hall, 1965).

Feyerabend, P.K., How to be a Good Empiricist, en Nidditch, P. (ed.) The Philosophy of Science, (Oxford: Oxford University Press, 1968).

Feyerabend, P.K., Consolidations for the Specialist, en Lakatos, I. and Musgrave, A. (eds.) Criticism and the Growth of Knowledge (Cambridge: Cambridge University Press, 1970).

Feyerabend, P.K. (ed.), Against Method (London: New Left Books, 1975).

Feyerabend, P.K., The Methodology of Scientific Research Programmes, en Feyerabend, P.K. (ed.) Problems of Empiricism, Philosophical Papers, Vol. 2 (Cambridge: Cambridge University Press, 1981).

Findlay, M.C. \& Williams, E.E., A Positivist Evaluation of the new Finance, Financial Management (1980) pp. 7-17.

Findlay, M.C. \& Williams, E.E., A Post-Keynesian View of Modern Financial Economics: In Search of Alternative Paradigms, Journal of Business Finance and Accounting (1985) pp. 1-18.

Freidson, E., Profession of Medicine (New York: Dodd, Mead, 1970).

Freidson, E., Professional Powers (University of Chicago Press, 1986).

Gordon, R.A. and Howell, J.E., Higher Education for Business (New York: Columbia University Press, 1959).

Hall, J.R., An Issue-oriented History of T.I.M.S., Interfaces (1983) pp. 9-19.

Harré, R., The Principles of Scientific Thinking (London: Macmillan, 1970).

Harré, R., Social Being (Oxford: Blackwells, 1979).

Hopwood, A., On Trying to Study Accounting in the Contexts in Which it Operates, Accounting Organizations and Society (1983) pp. 287-305.

Hopwood, A. \& Bromwich, M., Accounting Research in the United Kingdom, en Hopwood, A. and Schreuder, H. (eds.) European Contributions to Accounting Research (Amsterdam: Free University Press, 1984).

Hutchison, T., On the History and Philosophy of Science and Economics, en Latsis, S. (ed.) Method and Appraisal in Economics (Cambridge University Press, 1976).

Hutchison, T., Our Methodological Crisis, en Wiles, P. and Routh, G. (eds.) Economics in Disarray (Oxford: Blackwells, 1984).

Jensen, M.C., Capital Markets: Theory and Evidence, Bell Journal of Economics and Management (1972) pp. 357-398.

Kaplan, R.S., The Role for Empirical Research in Management Accounting, Accounting, Organizations and Society (1986) pp. 429-452.

Karpik, L., Organizations, Institutions and History, en Karpik, L. (ed.) Organization and Environment (London: Sage, 1978).

Kay, N.. The Emergent Firm (London: Macmillan, 1984). 
Kevles, D., The Physicist (New York: Alfred A. Knopf, 1977).

Knorr, K., Krohn, R. and Whitley, R. (eds.), The Social Process of Scientific Investigation Sociology of the Sciences Yearbook 4 (Dordrecht: Reidel, 1981).

Lakatos, I., Falsification and the Methodology of Scientific Research Programmes, en Lakatos, I. and Musgrave, A. (eds.) Criticism and the Growth of Knowledge (Cambridge University Press, 1970).

Lakatos, I., History of Science and its Rational Reconstructions, en Buck, R. and Cohen, R. (eds.) Boston Studies in the Philosophy of Science 8 (Dordrecht: Reidel, 1971).

Larson, M.S., The Rise of Professionalism (University of California Press, 1977).

Levy, R.M., The Professionalization of American Architects and Civil Engineers, Tesis de PhD no publicada, University of California at Berkeley (1980).

Locke, R.R., The End of the Practical Man: Entrepreneurship and Higher Education in Germany, France and Great Britain, 1880-1940 (Greenwich, CT: JAI Press, 1984).

Lowe, E.A., Puxty, A.G. \& Laughlin, R.C., Simple Theories for Complex Processes: Accounting Policy and the Market for Myopia, Journal of Accounting and Public Policy (1983) pp. 19-42.

Macdonald, K.M., Social Closure and Occupational Registration, Sociology (1985) pp. 541-556.

Mattessich, R., Methodological Preconditions and Problems of a General Theory of Accounting, Accounting Review (1972) pp. 469-487.

Merchant, C., The Death of Nature (New York: Harper and Row, 1980).

Musgrave, A., Unreal Assumptions in Economic Theory: The F-Twist Untwisted, Kyklos (1981), pp. 377-387.

Peasnell, K.V. \& Williams, D.J., Ersatz Academics and Scholar-Saints: the Supply of Financial Accounting Research, Abacus (1986) pp. 121-135.

Pierson, F.C., et al., The Education of American Businessmen (New York: McGraw-Hill, 1959).

Popper, K., Conjectures and Refutations (London: Routledge and Kegan Paul, 1963).

Popper, Karl, The Logic of Scientific Discovery (London: Hutchinson, 1968).

Pinch, Trevor, Confronting Nature (Dordrecht: Reidel, 1986).

Prigogine, I. \& Stengers, I., Order out of Chaos (London: Heinemann, 1984).

Reich, L.S., The Making of American Industrial Research (Cambridge University Press, 1985).

Ross, S., The Current Status of the Capital Asset Pricing Model, Journal of Finance (1978) pp. 885-901.

Ryan, R.J., Capital Market Theory - A Case Study in Methodological Conflict, Journal of Business Finance and Accounting (1982) pp. 443-458.

Schreuder, H., Positively Normative (Accounting) Theories, en Hopwood, A. and Schreuder, H. (eds.) European Contributions to Accounting Research (Amsterdam: Free University Press, 1984).

Seely, B.E., The Scientific Mystique in Engineering, Highway research at the Bureau of Public Roads 1918-1940, Technology and Culture (1984) pp. 798-831.

Stockman, N., Antipositivist Theories of the Sciences (Dordrecht: Reidel, 1983). 
Tinker, T., Paper Prophets (New York: Praeger, 1985).

Thomas, D., Naturalism and Social Science (Cambridge University Press, 1979).

Thomas, T.T., Towards a Value-neutral Positive Science of Accounting, Journal of Business Finance and Accounting (1981) pp. 549-572.

Tomkins, C. \& Groves, R., The Everyday Accountant and Researching his Reality, Accounting, Organizations and Society (1983) pp. 361-374.

Turner, S.P., Sociological Explanation as Translation (Cambridge University Press, 1980).

Watts, R.L. \& Zimmerman, J.L., Positive Accounting Theory (Englewood Cliffs, NJ: Prentice-Hall, 1986).

Whitley, R., The Fragmented State of Management Studies, Reasons and Consequences, Journal of Management Studies (1984) pp. 331-348.

Whitley, R., The Transformation of Business Finance into Financial Economics: The Roles of Academic Expansion and Changes in U.S. Capital Markets, Accounting Organizations and Society (1986) pp. 171-192.

Weber, M., The Methodology of the Social Sciences (New York: Free Press, 1949).

Wiebe, R.H., The Search for Order, 1877-1920 (New York: Hill and Wang, 1967).

Williamson, Oliver E., Markets and Hierarchies (New York: Free Press, 1975).

Wynne, Brian, C.G., Barkla and the J. Phenomenon, Social Studies of Science (1976) pp. 307-347.

\section{Políticas y normas contables}

\section{en una muestra de sociedades anónimas del Valle del Cauca}

ACUÑA M. Henao H. Prácticas Contables en el Valle del Cauca. Revista Cuadernos de Administración, No. 11 Univalle 1985, pp. 39-53.

BARONA B., Burbano J. y Sinisterra G. Programa de Investigación en Información Financiera Pública, Mimeo. Univalle 1987.

BALLESTEROS Enrique. Teoría y Estructura de la Nueva Contabilidad. Alianza Universidad Textos. Madrid 1979.

BALL Ray y FOSTER George. Corporate Financial Reporting. A Methodological Review of Empirical Research. Vol 20. USA 1982.

BELKAOUI Ahmed. Accounting Theory Editorial H.B.J. Inc. New York 1981.

BEAVER William. Financial Reporting and Accounting Revolution. Editorial PHI. New Jersey 1981.

BLANCO L. Yanel. Las normas de Contabilidad en Colombia. Edit. Roesga 1987.

BRIONES Guillermo. La Formulación de Problemas de Investigación Social. Ediciones Uniandes. Bogotá 1981.

BRUNS y De Coster D. La Contabilidad y el Comportamiento Humano. Editorial Trillas: México 1975. 
CARDONA John. Estructura Básica de la Contabilidad para Colombia. Revista No. 14 Contaduría Universidad de Antioquia. Medellín 1989, pp. 43-111.

CARDONA John. La Revelación Contable - Un Estudio Exploratorio. Revista No. 8 Universidad de Antioquia. Medellín 1986, pp 77-111.

Cámara de Comencio de Cali. Boletín Estadístico sobre Inversión Privada en el Valle del Cauca. Cali 1986.

Cámara de Comercio de Bogotá. Decreto 2160 de 1986.

CAÑIBANO Leandro. Teoría Actual de la Contabilidad. Biblioteca de Ciencias Empresariales Ediciones ICE. Madrid 1979.

GEORGE Claude. Historia del Pensamiento Administrativo Editorial PHI. México 1984.

HANAN Mack. Incremento de utilidades. Cómo acelerarlo mediante la aplicación de estrategias empresariales. Editorial Norma. Bogotá 1982.

HENDRICKSEN Eldon. Teoría de la Contabilidad. Edit. Uteha. México 1981.

HORNGREN Charles. Contabilidad Administrativa Introducción. Edit PHI. Carvajal. Cali 1983.

KAST. F. y ROSENZWEIG J. Administración en las Organizaciones. Un enfoque de Sistemas. Edit. Mc Graw Hill. México 1980.

MATTESICH Richard. Un examen científico aplicado para una estructura metodológica. Revista Teuken No. 3. C. Rivadavia. Argentina 1988.

MCNICHOLS Thomas. Política Empresarial. Edit. Mc Graw Hill. Bogotá 1981.

MLLER Martin. Guía de Principios de Contabilidad generalmente aceptados. Editorial H.B.J. New York 1982.

MORRISSEY Leonard. Teoría Contable de la Información Financiera. Editorial Trillas. México 1977.

LEE Thomas. Developments in Company Financial Reporting a History and an Introduction. Philip Alan Publishers Limited. Oxford 1981.

LIBBY Robert. Accounting and Human Information Processing: Theory and Aplications. Edit. Prentice Hall Inc. Englewood Cliffs. New Jersey 1981.

PARDINAS Felipe. Metodología y Técnicas de Investigación en Ciencias Sociales. Editorial "Siglo XXI Editores". México 1983.

RODRIGUEZ Mario. La información contable y su exposición. Administración de Empresas. Tomo II.

SHIM J. y SIEGEL J. Contabilidad Administrativa - Ser ie Schaum. Editorial Mc Graw Hill. Bogotá 1986.

SIMKIN M. y MOSCOVE Stephen. Accounting Information Systems Concepts and practice for effective decision. Makin Edit. John Wiley y Sons Inc. New York 1979.

TUA Pereda Jorge. Principios y Normas de Contabilidad, impreso en la Fábrica Nacional de Moneda y Timbre. Madrid 1983.

UDUAL. IV Conferencia de Facultades y Escuelas de Contaduría Pública de América Latina - III Congreso Latinoamericano de Investigación Contable. Universidad Central. Bogotá 1986. 
VASQUEZ José. Contabilidad Moderna. Edit. Bedout. Medellín 1947.

VLAEMMINCK Joseph. Historia y doctrinas contables. Editorial Index. Madrid 1961.

WATTZ y ZIMMERMAN. Positive Accounting Theory. Editorial PHI. New Jersey 1986.

ZEFF Stephen. Evolución de la Teoría Contable. La investigación empírica. Revista Contaduría Universidad de Antioquia. No. 6 Marzo de 1985, pp 25-53. 